\title{
ANÁLISE DAS HABILIDADES AUDITIVAS DE CRIANÇAS COM RESPIRAÇÃO ORAL
}

\author{
Analysis of hearing abilities in mouth-breathing children
}

\author{
Bruna Machado Correa ${ }^{(1)}$, Angela Garcia Rossi ${ }^{(2)}$, Bruna Roggia ${ }^{(3)}$, Ana Maria Toniolo da Silva ${ }^{(4)}$
}

\begin{abstract}
RESUMO
Objetivo: destacar possíveis relações entre respiração oral e o sistema auditivo central de crianças em idade escolar, a partir da análise das habilidades auditivas de resolução temporal integração e separação binaural, atenção dividida, memória auditiva e fechamento auditivo. Método: estudo em 102 escolares, entre 8 e 12 anos. Grupo I composto por 52 crianças com respiração oral e 50 crianças formaram o grupo II, com respiração nasal. Foram utilizados testes de Fala Filtrada, Teste de Padrões de Freqüência e Dicótico de Dissílabos Alternados (SSW). Os resultados dos testes foram analisados em relação ao tipo de habilidade alterada e sua ocorrência em crianças com respiração oral. Além de uma análise quanto à possível relação entre os testes e as ocorrências de alterações mais freqüentes entre os mesmos. Resultados: indivíduos do grupo I obtiveram maior freqüência de alterações de grau severo e moderado. Comprovou-se diferença estatisticamente significante entre os grupos para as condições de: orelhas esquerda e direita competitiva, alteração relacionada a organização e integração auditiva, fechamento auditivo e padrão temporal. Não ocorreu diferença estatisticamente significante nos resultados da relação entre testes. Conclusão: crianças com respiração oral apresentam desempenho inferior nas habilidades do processamento auditivo do que crianças com padrão respiratório normal.
\end{abstract}

DESCRITORES: Audição; Percepção Auditiva; Respiração Bucal; Transtornos da Percepção Auditiva; Aprendizagem

\section{INTRODUÇÃO}

O sistema nervoso auditivo central é um sistema de vias neurais complexas, que pode ser afetado por diversos fatores desenvolvimentais e patológicos.

O processamento auditivo é um conjunto de habilidades auditivas que o indivíduo necessita

(1) Fonoaudióloga; Mestre em Distúrbios da Comunicação Humana pela Universidade Federal de Santa Maria (UFSM), Santa Maria, Rio Grande do Sul, Brasil.

(2) Fonoaudióloga; Doutora em Distúrbios da Comunicação Humana, Professora Adjunta do Curso de Distúrbios da Comunicação Humana da Universidade Federal de Santa Maria (UFSM), Santa Maria, Rio Grande do Sul, Brasil.

(3) Fonoaudióloga; Mestre em Distúrbios da Comunicação Humana pela Universidade Federal de Santa Maria (UFSM), Santa Maria, Rio Grande do Sul, Brasil.

(4) Fonoaudióloga; Doutora em Distúrbios da Comunicação Humana, Professora Adjunta do Curso de Distúrbios da Comunicação Humana da Universidade Federal de Santa Maria (UFSM), Santa Maria, Rio Grande do Sul, Brasil.

Conflito de interesses: inexistente para interpretar o que ouve. ${ }^{1}$,as quais capacitam o som, que após ser detectado pela orelha interna, sofre inúmeros processos cognitivos e fisiológicos para que ocorra a decodificação e compreensão do mesmo².

Distúrbios do processamento auditivo resultam de disfunções dos processos dedicados a audição.

As alterações comportamentais encontradas em crianças com respiração oral podem demonstrar uma possível alteração do processamento auditivo, pela semelhança entre as mesmas. A respiração é vital para o organismo, sendo importante para o bom funcionamento de todo o ser humano. Através da via nasal ocorre a função respiratória normal, no entanto, quando ocorre alguma obstrução das vias respiratórias e/ou algum problema funcional, a respiração pode ser alterada para oral.

Entre as alterações comportamentais encontradas em crianças com respiração oral, destacamse o sono agitado, a irritabilidade, a dificuldade de concentração acompanhada de queda no 
rendimento escolar e da baixa aptidão esportiva, entre outras ${ }^{3}$. A sonolência diurna pode interferir na atenção da criança, prejudicando o aprendizado ${ }^{4}$.

A alteração no sistema hematológico do respirador oral interfere no raciocínio, no humor, na saúde geral, podendo levar a uma deterioração da qualidade de vida e ao envelhecimento precoce deste ${ }^{5}$.

O indivíduo com Desordem do Processamento Auditivo pode apresentar algumas queixas associadas, como a existência de problemas respiratórios concomitantes levando a respiração oral.

Sabe-se da existência da relação entre as conseqüências da respiração oral e o processamento auditivo. Crianças em idade escolar com respiração oral apresentam queixas de dificuldades escolares e de alfabetização, assim como crianças que apresentam alteração do processamento auditivo.

O presente trabalho teve com objetivo estudar as possíveis relações entre a respiração oral e o processamento auditivo, a partir de uma análise das habilidades auditivas de resolução temporal (reconhecimento dos padrões de freqüência, ordenação temporal e nomeação), integração e separação binaural, atenção dividida, memória auditiva e fechamento auditivo. Além de uma análise entre os testes realizados, comparando seus resultados entre si.

\section{MÉTODO}

Esta pesquisa é um estudo transversal, descritivo, contemporâneo e quantitativo de dados obtidos no Laboratório de Otologia do Hospital Universitário de Santa Maria (HUSM), vinculado a Universidade Federal de Santa Maria (UFSM). A pesquisa foi realizada em uma escola da rede municipal da cidade de Santa Maria.

Obedecendo à Resolução 196/1996 da CONEP, participaram deste estudo os sujeitos que assentiram participar da pesquisa e cujos pais ou responsáveis assinaram o TCLE, após terem sido informados sobre o objetivo e a metodologia do estudo proposto.

A amostra foi composta 102 crianças escolares, com idade entre 8 e 12 anos, normo-ouvintes que apresentaram diagnóstico fonoaudiológico de respiração oral. Foram avaliadas 52 crianças com diagnóstico fonoaudiológico de respiração oral (grupo I) e 50 com respiração nasal (grupo II). Destes, 49 eram do gênero masculino e 53 do gênero feminino.

Foram excluídos da amostra os indivíduos com perda auditiva do tipo neurossensorial, condutiva e/ ou mista, de qualquer grau, alterações neurológicas, motoras, mentais ou outra alteração que pudesse prejudicar a compreensão e realização das tarefas propostas e estar em tratamento ortodôntico ou fonoaudiológico na área de motricidade oral.

Juntamente com o TCLE foram entregue a todos os pais e/ou responsáveis fichas de anamnese contendo 65 perguntas que envolviam dados pessoais, desenvolvimento neuropsicomotor, história clínica de doenças, cirurgias e tratamentos, características pessoais, saúde geral, presença de hábitos deletérios, distúrbios do sono, rendimento escolar, entre outros. Foi solicitado também, que os professores observassem as crianças constituintes de suas classes e encaminhassem para a avaliação fonoaudiológica aquelas que suspeitaram de ser respiradora oral.

Foram considerados para definição do grupo I:

- Existência de queixas e manifestações clínicas mais comuns em quadros de respiração oral, sendo que nas anamneses em que foram encontrados três ou mais queixas de respiração oral as crianças foram consideradas como constituintes do grupo I. Já para a formação do grupo II foram consideradas as anamneses em que menos de três características de respiração oral foram encontradas ${ }^{6}$.

- Avaliação do sistema estomatognático, incluindo teste da água, para avaliar o tempo de permanência de água na boca com os lábios em contato e sem engolir até três minutos cronometrados.

- Avaliação audiológica básica: inspeção visual do meato acústico externo; audiometria tonal liminar por via aérea nas freqüências de 250 a $8.000 \mathrm{~Hz}$ e por via óssea nas freqüências de 500 a $4.000 \mathrm{~Hz}$; pesquisa do limiar de reconhecimento de fala (LRF); pesquisa do índice percentual de reconhecimento de fala (IPRF); Imitânciometria (curvas timpanométricas e reflexos acústicos).

A avaliação do Processamento Auditivo foi realizada a partir do Teste de Fala Filtrada (avaliar fechamento auditivo), do Teste de Padrões de Freqüência (avaliar reconhecimento dos padrões de freqüência, ordenação temporal e nomeação) e do Teste Dicótico de dissílabos Alternados - SSW (avaliar integração e separação binaural, atenção dividida e memória).

No Teste de Fala Filtrada o esperado para a idade são valores maiores ou iguais a $70 \%$ de acertos.

O Teste de Padrões de Freqüência (TPF) tem como resultado esperado para crianças entre $8 \mathrm{e}$ 9 anos, valor maior ou igual a $91 \%$ de acertos. $\mathrm{E}$ para crianças acima de 9 anos, valor maior ou igual a $76 \%$. 
Para o Teste Dicótico de dissílabos Alternados SSW foi realizada análise quantitativa, utilizando a média das condições competitivas (DC e EC). E análise qualitativa, realizada quanto às tendências das respostas, que são: Inversões, efeito auditivo, efeito de ordem e padrão de resposta tipo $A$.

As medidas desta pesquisa foram obtidas em cabina tratada acusticamente, utilizando um audiômetro digital de dois canais, marca Fonix, modelo FA-12, tipo I e fones auriculares tipo TDH-39P, marca Telephonics. Os testes do processamento auditivo foram apresentados utilizando-se um Compact Disc Player Digital Toshiba - 4149, acoplado ao audiômetro descrito acima.

Os resultados dos testes foram analisados a partir da relação entre o tipo de habilidade alterada e sua ocorrência em crianças com diagnóstico fonoaudiológico de respiração oral. Além de uma análise quanto à possível correlação entre os testes FF, TPF e SSW e as ocorrências de alterações mais freqüentes entre os mesmos.

A pesquisa foi realizada a partir da aprovação do projeto pelo Comitê de Ética da instituição de origem, sob o protocolo número 23081.0017140/2008-25.

Para investigar o nível de associação entre os resultados obtidos foi aplicado o método estatístico Qui-quadrado e Teste de Ficher. Considerou-se o nível de significância estatística de $p \leq 0.05$.

\section{RESULTADOS}

A Tabela 1 permite a visualização das ocorrências de alterações, classificadas em Subperfis do Processamento auditivo, nos grupos estudados (I e II).

O Subperfil de Decodificação Fonêmica ocorreu em $26,96 \%$ dos indivíduos do grupo I e em 34,00\% dos indivíduos do grupo II. O Subperfil denominado Perda gradual de memória ocorreu em $46,15 \%$ indivíduos do grupo I e em 58,00\% indivíduos do grupo II. O Subperfil de Organização ocorreu em 32,69\% indivíduos do grupo I e em $10,00 \%$ indivíduos do grupo II. Quanto ao Subperfil de Integração ocorreu em $53,85 \%$ indivíduos do grupo I e em $26,00 \%$ indivíduos do grupo II.

Na Tabela 2 é apresentada a relação das alterações referentes ao grau de severidade encontradas no Teste Dicótico de dissílabos Alternados - SSW, entre os grupos I e II.

Nesta tabela destaca-se: os indivíduos do grupo I obtiveram maior freqüência de alterações de grau severo e moderado (ambos $30,77 \%$ ), seguido de alterações de graus leve $(21,15 \%)$ e uma pequena porcentagem de crianças sem alteração $(17,31 \%)$. Os indivíduos do grupo II apresentaram maior freqüência de resultados normais $(54,00 \%)$, seguido de alterações de grau leve $(30,00 \%)$ e moderado (16,00\%), sendo que alterações de grau severo não ocorreram neste grupo.

Tabela 1 - Classificação dos Subperfis de alteração do Processamento Auditivo

\begin{tabular}{ccccc}
\hline & DF (n) & PGM (n) & O (n) & I (n) \\
\cline { 2 - 5 } & Alt & Alt & Alt & Alt \\
\hline Grupo I & $14 / 52(26,96 \%)$ & $24 / 52(46,15 \%)$ & $17 / 52(32,69 \%)$ & $28 / 52(53,85 \%)$ \\
Grupo II & $17 / 50(34,00 \%)$ & $29 / 50(58,00 \%)$ & $5 / 20(10,00 \%)$ & $13 / 50(26,00 \%)$ \\
p-value & 0.4373 & 0.2313 & 0.0053 & 0.0041 \\
\hline
\end{tabular}

DF - Decodificação Fonêmica; PGM - Perda Gradual de Memória; O - Organização; I - Integração; n - número de sujeitos; Alt - resultados alterados.

Valor de $\mathrm{p}<0.05$ indica diferença estatisticamente significativa

Tabela 2 - Resultados do Teste SSW referente ao grau de severidade em ambos os grupos

\begin{tabular}{ccccccc}
\hline & & Leve & Moderado & Normal & Severo & Total \\
\hline \multirow{2}{*}{ Grupo I } & $\mathrm{n}$ & 11 & 16 & 9 & 16 & 52 \\
& $\%$ & 21.15 & 30.77 & 17.31 & 30.77 & 50.98 \\
\hline \multirow{2}{*}{ Grupo II } & $\mathrm{n}$ & 15 & 8 & 27 & 0 & 50 \\
& $\%$ & 30.00 & 16.00 & 54.00 & 0.00 & 49.02 \\
\hline \multirow{2}{*}{ Total } & $\mathrm{n}$ & 26 & 24 & 36 & 16 & 102 \\
& $\%$ & 25.49 & 23.53 & 35.29 & 15.69 & 100.00 \\
\hline
\end{tabular}

SSW - Teste dicótico de dissílabos alternados; $\mathrm{n}$ - número de sujeitos. 
$\mathrm{Na}$ Tabela 3 são apresentados os resultados encontrados no teste SSW para direita e esquerda competitiva nos grupos I e II.

Entre os dois grupos é possível verificar que os percentuais de alterações das habilidades do processamento auditivo que o teste SSW avalia foram correspondentemente mais elevados entre as crianças com diagnóstico fonoaudiológico de respiração oral do que das com respiração nasal para as condições de: orelha esquerda e orelha direita competitiva, graus de severidade, alteração relacionada à organização e integração auditiva. A partir dos testes estatísticos realizados comprovou-se diferença estatisticamente significante entre os grupos estudados, ao nível de $5.0 \%$, para as condições relacionadas acima. Em relação à presença de alteração em Decodificação Fonêmica e Perda Gradual de Memória, não houve diferença estatisticamente significante entre os grupos.

Tabela 3 - Resultados quantitativos do teste SSW para ambos os grupos

\begin{tabular}{cccccc}
\hline & \multicolumn{3}{c}{ DC (n) } & \multicolumn{3}{c}{ EC (n) } \\
\cline { 2 - 6 } & Alterado & Normal & Alterado & Normal \\
\hline Grupo I & 29 & & 23 & 41 & 11 \\
Grupo II & 13 & & 37 & 20 & 30 \\
$p$-value & & 0.0023 & & & $<0.0001$ \\
\hline
\end{tabular}

SSW - Teste dicótico de dissílabos alternados; DC - Direita Competitiva; EC - Esquerda Competitiva.

Valor de $p<0.05$ indica diferença estatisticamente significativa

Na Tabela 4 são apresentados os resultados do teste de FF, em ambas as orelhas, realizados nos grupo I e II.
Desta tabela verifica-se que houve diferença estatisticamente significante $(p<0.05)$ entre o grupo com respiração oral (I) e com respiração nasal (II), tanto na orelha direita quanto na orelha esquerda.

Tabela 4 - Resultados do teste FF para ambos os grupos

\begin{tabular}{cccccc}
\hline & \multicolumn{2}{c}{ OD $(\mathbf{n})$} & & \multicolumn{2}{c}{ OE $(\mathbf{n})$} \\
\cline { 2 - 6 } & Alterado & Normal & Alterado & Normal \\
\hline Grupo I & 27 & 25 & 27 & 25 \\
Grupo II & 1 & 49 & 1 & 49 \\
p-value & & $<0.0001$ & & & $<0.0001$ \\
\hline
\end{tabular}

FF - Fala Filtrada; OD - orelha direita; OE - Orelha esquerda; - número de sujeitos.

Valor de $p<0.05$ indica diferença estatisticamente significativa

A Tabela 5 apresenta os resultados encontrados no teste TPF, na forma nomeada e murmurada em ambas às orelhas, para os dois grupos pesquisados.

Percebe-se que neste teste também ocorreu diferença estatisticamente significante entre os grupos I e II $(p<0.05)$, tanto na forma murmurada quanto na nomeada, demonstrando uma maior ocorrência de alterações das habilidades de padrão temporal em crianças com respiração oral.

Ao ser aplicado o teste de Qui-quadrado para os dados obtidos, verificou-se que o valor de significância estatística do mesmo foi menor que 5\% também nos testes de FF e TPF. Por isso, a relação entre o desempenho nos testes do processamento auditivo, nas habilidades de fechamento auditivo e padrões temporais da audição, e o tipo de respiração foi considerado como estatisticamente significante, ou seja, desempenho nestes testes do processamento auditivo foi associado ao tipo de respiração.

As Tabelas 6 e 7 apresentam a análise da relação entre as respostas de cada teste (FF, TPF e SSW) e as ocorrências de alterações mais freqüentes entre os mesmos. Tal relação não apresentou diferença estatisticamente significante. 
Tabela 5 - Resultados do teste TPF para ambos os grupos

\begin{tabular}{cccccccccc}
\hline & \multicolumn{2}{c}{ OD nomeado (n) } & \multicolumn{2}{c}{ OD murmurado $(\mathbf{n})$} & \multicolumn{2}{c}{ OE nomeado $(\mathbf{n})$} & \multicolumn{2}{c}{ OE murmurado $(\mathbf{n})$} \\
\cline { 2 - 9 } & Alterado & Normal & Alterado & Normal & Alterado & Normal & Alterado & Normal \\
\hline Grupo I & 29 & 23 & 22 & 30 & 30 & 22 & 26 & 26 \\
Grupo II & 14 & 36 & 10 & 40 & 18 & 32 & 10 & 40 \\
$p$-value & \multicolumn{2}{c}{0.0045} & \multicolumn{2}{c}{0.0152} & \multicolumn{2}{c}{0.0282} & 0.0015 \\
\hline
\end{tabular}

TPF - Teste de Padrões de Frequencia; OD - orelha direita; OE- orelha esquerda, n- número de sujeitos.

Valor de $p<0.05$ indica diferença estatisticamente significativa

Tabela 6 - Relação entre testes no grupo I

\begin{tabular}{|c|c|c|c|c|c|}
\hline \multicolumn{2}{|c|}{ Grupo I } & \multicolumn{2}{|c|}{ TPF } & \multicolumn{2}{|c|}{ FF } \\
\hline & & Alterado (n) & Normal (n) & Alterado (n) & Normal (n) \\
\hline \multirow{3}{*}{ SSW } & Alterado & 28 & 16 & 23 & 21 \\
\hline & Normal & 3 & 5 & 6 & 2 \\
\hline & $p$-value & \multicolumn{2}{|c|}{0.1215} & \multicolumn{2}{|c|}{0.1597} \\
\hline \multirow{3}{*}{ TPF } & Alterado & & & 19 & 12 \\
\hline & Normal & & & 10 & 11 \\
\hline & $p$-value & & & \multicolumn{2}{|c|}{0.1411} \\
\hline
\end{tabular}

FF - Fala Filtrada; TPF - Teste de padrões de Freqüência; SSW - Teste Dicótico de Dissílabos Alternados; $n$ - número de sujeitos. Valor de $\mathrm{p}<0.05$ indica diferença estatisticamente significativa

Tabela 7 - Relação entre testes no grupo II

\begin{tabular}{|c|c|c|c|c|c|}
\hline \multirow{2}{*}{\multicolumn{2}{|c|}{ Grupo II }} & \multicolumn{2}{|c|}{ TPF } & \multicolumn{2}{|c|}{ FF } \\
\hline & & Alterado (n) & Normal (n) & Alterado (n) & Normal (n) \\
\hline \multirow{3}{*}{ SSW } & Alterado & 12 & 18 & 1 & 29 \\
\hline & Normal & 6 & 14 & 0 & 20 \\
\hline & $p$-value & \multicolumn{2}{|c|}{0.1857} & \multicolumn{2}{|c|}{0.6000} \\
\hline \multirow{3}{*}{ TPF } & Alterado & & & 0 & 18 \\
\hline & Normal & & & 1 & 31 \\
\hline & $p$-value & & & \multicolumn{2}{|c|}{0.6400} \\
\hline
\end{tabular}

FF - Fala Filtrada; TPF - Teste de padrões de Freqüência; SSW - Teste Dicótico de Dissílabos Alternados; n- número de sujeitos. Valor de $p<0.05$ indica diferença estatisticamente significativa

Quanto aos resultados dos indivíduos do grupo I, relacionando os testes SSW e TPF, 53,85\% tiveram alteração nos dois testes, 30,77\% tiveram alteração do SSW e TPF normal, 5,77\% tiveram SSW normal e TPF alterado e 9,62\% tiveram resultados normais para os dois testes $(p=0.1215)$. Já os indivíduos do grupo II apresentaram: $24,00 \%$ resultados alterados nos dois testes, $36,00 \%$ com SSW alterado e TPF normal, $12 \%$ com SSW normal e TPF alterado e $28,00 \%$ com resultados normais para os dois testes $(p=0.1857)$.

Na realização da associação entre testes SSW e FF, os indivíduos do grupo I apresentaram: $44,23 \%$ resultados alterados nos dois testes, $40,38 \%$ com
SSW alterado e FF normal, $11,54 \%$ com SSW normal e FF alterado e $3,85 \%$ com resultados normais para os dois testes ( $p=0.1597)$. Já os indivíduos do grupo II apresentaram: $2 \%$ alteração nos dois testes, 58,00\% alteração do SSW e FF normal, $0,00 \%$ SSW normal e FF alterado e $40,00 \%$ resultados normais para os dois testes $(p=0.6000)$.

Quanto aos indivíduos do grupo I, relacionando os testes TPF e FF, 36,54\% apresentaram alteração nos dois testes, $23,08 \%$ apresentaram alteração do TPF e FF normal, 19,23\% apresentaram TPF normal e FF alterado e $21,15 \%$ apresentaram resultados normais para os dois testes $(p=0.1411)$. Quanto aos indivíduos do grupo II: nenhum 
apresentou resultados alterados nos dois testes, $36 \%$ obtiveram resultados do TPF alterado e FF normal, $2 \%$ obtiveram resultados TPF normal e FF alterado e $62 \%$ obtiveram resultados normais para os dois testes $(p=0.6400)$.

\section{DISCUSSÃO}

No Brasil, o termo processamento auditivo é utilizado para designar a série de processos que envolvem estruturas do sistema nervoso central: vias auditivas e córtex ${ }^{7}$. Tal habilidade auditiva tem papel fundamental no desenvolvimento da linguagem e da fala.

A desordem do processamento auditivo é considerada como sendo um distúrbio da audição, no qual há um impedimento da habilidade de analisar e/ou interpretar padrões sonoros ${ }^{7}$.

Já em relação a respiração oral, na elaboração do diagnóstico de um problema respiratório é importante a informação que o paciente fornece ao profissional durante a anamnese, pois a mesma, dependendo da duração, pode causar diversas alterações.

No teste SSW, quanto aos Subperfis de Decodificação fonêmica e Perda Gradual de Memória, não houve diferença estatisticamente significante entre os grupos I e II, sendo que os mesmos seguiram os padrões de normalidade descritos na literatura ${ }^{7}$, não sendo observada relação entre as crianças respiradoras orais e possíveis alterações das habilidades de decodificação fonêmica e memória auditiva.

Foram encontrados, no presente estudo, graus de severidade em maior ocorrência, de moderado a severo, no grupo de crianças com respiração oral. Esta relação pode ser explicada pelo baixo escore em atividades de atenção e raciocínio que crianças com respiração oral apresentam na realização de tarefas em que são necessárias estas habilidades ${ }^{5}$. Segundo a autora a alteração no sistema hematológico do respirador oral interfere no raciocínio, no humor e na saúde geral.

Testes de escuta dicótica, como o SSW, são utilizados para avaliar o processamento neurológico de informações auditivas recebidas pelo indivíduo.

A presença de alterações no teste SSW, relacionadas às condições de: orelha esquerda e orelha direita competitiva, alteração relacionada à organização e integração auditiva, também mostraram diferença estatisticamente significante entre os grupos, o que é explicado como decorrente das alterações comportamentais e da diminuição da oxigenação cerebral presente nestas crianças. Estes dados corroboram com estudo que mostra que as alterações encontradas nas crianças com respi- ração oral podem ocorrer pela insuficiente irrigação sanguínea cerebral, devido ao padrão respiratório ${ }^{4}$.

O autor ainda refere que entre as alterações do respirador oral, a sonolência diurna pode interferir na atenção da criança, prejudicando assim o aprendizado.

A desordem do processamento auditivo esta relacionada com distúrbios de aprendizagem e déficits de atenção, destacando que estas são entidades clínicas distintas, porém com possibilidades de coexistência 9 .

A partir da análise dos resultados pode-se verificar que há uma relação existente entre as alterações encontradas nas crianças com respiração oral nas habilidades auditivas de ordenação temporal e de fechamento auditivo, por apresentar resultados abaixo do esperado para a idade nos testes TPF e FF.

Crianças com respiração oral apresentam dificuldade de memorizar o que foi apresentado. Esta dificuldade decorre das alterações comportamentais apresentadas por estas crianças, confirmando os dados obtidos nesta pesquisa.Em estudo recente os autores referem-se à memória como fazendo parte das habilidades auditivas, que é uma das funções do sistema nervoso, e pode ser definida como a aquisição (ou aprendizado), o armazenamento e a evocação de informações ${ }^{10}$.

A maioria das informações que constitui a memória é aprendida pelos sentidos em episódios que são denominados experiências, havendo tantas memórias possíveis quanto o número de experiências. Ao avaliar o processamento de informações recebidas por meio da audição, também é possível observar como o indivíduo utiliza o seu sistema de gerenciamento de informações, denominado memória operacional ou de trabalho ${ }^{10}$.

A atenção sustentada e a vigilância são alguns dos processos que caracterizam a atenção e são fundamentais para o desenvolvimento de algumas habilidades ${ }^{11}$.

Em estudo recente sobre a influência de padrões temporais no processamento auditivo, nos testes envolvendo freqüência, em relação à ordem solicitada, o estudo mostra pior desempenho das crianças nas tarefas de ordenação, se comparada com discriminação dos sons. Referem que variáveis temporais como duração do estímulo e tipo de ordem solicitada (discriminação e ordenação) podem interferir no desempenho de crianças em testes de processamento temporal auditivo ${ }^{12}$.

O processamento auditivo e a resolução temporal são fundamentais para o desenvolvimento da linguagem ${ }^{13}$. Esta habilidade esta correlacionada positivamente com habilidades de complexidade sintática, sendo que o baixo desempenho em 
teste de padrões de freqüência pode servir como indicativo de déficits em processamento lingüístico complexo ${ }^{14}$.

A avaliação do processamento auditivo, neste estudo, foi realizada por meio de testes comportamentais. Esses são considerados como a principal chave no diagnóstico de alterações de processamento auditivo em adultos e crianças.

Os resultados do presente estudo são indicadores de que crianças respiradoras orais apresentam desempenho pior nos testes do processamento auditivo, por apresentar dificuldades de atenção e concentração, como descritos por vários autores como uma de suas características ${ }^{15}$.

\section{CONCLUSÃO}

Os resultados encontrados neste trabalho permitiram concluir que:

- Crianças com respiração oral apresentam desempenho inferior nas habilidades do processamento auditivo do que crianças com padrão respiratório normal.

- A avaliação do processamento auditivo não mostrou associação entre os resultados dos diferentes testes.

\begin{abstract}
Purpose: to highlight the possible relationships between mouth breathing and central auditory system of school-age children, based on an analysis of the auditory abilities of temporal resolution, binaural separation and integration, divided attention, auditory memory and closure. Method: a study of 102 school children, between 8 and 12-year old. Group I was composed of 52 children with mouth breathing and group II was composed of 50 children with nose breathing. The evaluation was carried out using Filtered Speech tests, Pitch Pattern Sequence and Alternating Staggered Spondaic Word. Results: subjects from Group I had higher frequency of severe and moderate alterations. A statistically significant difference occurred between the groups for the left and right competitive ear, changes related to organization and auditory integration, auditory closure abilities and temporal patterns. There was no statistically significant difference in the relationship among the tests. Conclusion: the performance on the tests for auditory processing was associated to the type of breathing.
\end{abstract}

KEYWORDS: Hearing; Auditory Perception; Mouth Breathing; Auditory Perceptual Disorder; Learning

\section{REFERÊNCIAS}

1. Russo ICP. A relevância da pesquisa científica na audiologia brasileira. Rev. CEFAC. 2009. Disponível em: $\quad$ http://www.scielo.br/scielo.php?pid=S1516$18462009000500002 \&$ script=sci_arttext\&tIng=en . Acesso em: 28/05/2010.

2. Ramos BD, Alvarez AM, Sanchez ML. Neuroaudiologia e processamento auditivo: novos paradigmas. RBM/ORL Controvérsias \& Interfaces.2007; 2(1): 51-8.

3. Queluz DP, Gimenez CMM. A síndrome do respirador bucal. Rev CROMG. 2000; 6(1): 4-9.

4. Di Francesco RC. Respirador bucal: a visão do otorrinolaringologista. J Bras Fonoaudiol. 1999; 1(1): 56-60.

5. Cintra CF, Castro FM, Morato FF, Cintra PP. As alterações oro-faciais apresentadas em pacientes respiradores bucais. Rev Bras Alergia Imunopatol. 2000; 23(1): 78-83.
6. Abreu RR, Rocha RL, Lamounier JL, Guerra AFM. Prevalence of mouth breathing among children. Jornal de Pediatria. 2008; 84(5): 467-70.

7. Pereira LD, Schochat E. Processamento Auditivo Central- abordagem passo a passo. In: Pereira LD, Schochat E. Processamento Auditivo CentralManual de Avaliação. São Paulo: Lovise, 1997.

8. Ríos AA, Rezende AG, Pela SM, Ortiz KZ, Pereira LD. Teste de padrão harmônico em escuta dicótica - TDDH. Rev Soc Bras Fonoaudiol. 2007; 12(4): 304-9.

9. Ferreira MIDC, Mello AM. Comorbidade entre transtorno de déficit de atenção e hiperatividade e distúrbio do processamento auditivo. Rev Fonoaudiol Brasil. 2006; 4(2): 1-3.

10. Izquierdo I, Vianna MRM, Camarotta AM, Izquierdo LA. Mecanismos de memória. Scientific American. 2003; 2(17): 98-104.

11. Feniman MR, Ortelan RR, Lauris JRP, Campos CF, Cruz MS. Proposta de instrumento 
comportamental para avaliar a atenção auditiva sustentada. Rev Bras de Otorrinolaringol. 2007; 73(4): 523-7.

12. Murphy CFB, Schochat E. Influência de paradigmas temporais em testes de processamento temporal auditivo. Pró-fono. 2007; 19(3): 259.

13. Samelli AG, Schochat E. Processamento auditivo, resolução temporal e teste de detecção de gap: revisão da literatura. Rev. CEFAC. 2008; 10(3): 369-77.
14. Fortunato-Tavares $\mathrm{T}$, Rocha $\mathrm{CN}$, Andrade CRF de, Befi-Lopes DM, Schochat E, Hestvik A, Schwartz RG. Processamento lingüístico e processamento temporal em crianças com distúrbio específico de linguagem. Pró-fono. 2009; 21(4):279-84.

15. Di Francesco RC, Chedid KA, Junqueira AS. Respiração oral afeta aprendizado. 2007. disponível em: <http://www.clicfilhos.com.br/site/home.htm>. Acesso em: 07/05/2009.

Endereço para correspondência:

Bruna Machado Correa

Rua Gaspar Martins, 98

Cachoeira do Sul - RS

CEP: 96501-500

E-mail: brucorrea_tunis@ $@$ hotmail.com 